\title{
To Study The Status of LH: FSH Ratio in Obese And Non-Obese Patients of Polycystic Ovarian Syndrome.
}

\author{
Laksman Lal ${ }^{1}$, Atima Bharti ${ }^{1}$, Arshi Perween ${ }^{2}$
}

\begin{abstract}
Aim : To evaluate LH: FSH ratio in obese and non-obese patients of Polycystic Ovarian Syndrome(PCOS).

Material and method:The study was conducted in Rajendra Institute of Medical Sciences, Ranchi. 102 patients were selected from patients visiting department of obstetrics and gynaecology, dermatology and medicine for complaint of menstrual irregularities, acne, hirsuitism, obesity and infertility. Ethical consent was taken. After proper counselling, informed consent of patients were taken. Fasting blood sample was taken on $3^{\text {rd }}$ day of normal or induced menstrual cycle. Serum Luteinizing Hormone (LH) and Follicular stimulating hormone (FSH) was estimated with the help of chemiluminescence ABBOTT 1000 isr.

Results: $78.43 \%$ had high level of LH. 72.5\% had low FSH level. 74.5\% had high LH: FSH ratio. 13.44\% obese patient had high LH compared to $12.22 \%$ of normal weight patient. $5.29 \%$ of obese patient had low FSH level compared to $5.86 \%$ of normal weight patient. Similarly $2.67 \%$ of obese patient had high LH: FSH ratio.

Conclusion: In PCOS women gonadotrophin ovarian axis is disturbed which is reflected by high LH and low FSH value. This hormonal imbalance leads to oligomenorrhoea, anovulation leading to infertility. Acne, hirshutism and obesity is seen in such patient of PCOS. Life style modification can help in curbing the disease to a great extent.
\end{abstract}

Keywords: Luteinizing Hormone (LH), Follicular stimulating hormone (FSH), Polycystic Ovarian Syndrome, Obesity.

\section{Introduction}

In recent year there have been changes in the life style, economic growth, and increased affluence of middle class, Urbanization, marked changes in diet and sedentary habits. All these have led to an increase in life style related disease. One such disease entity is Polycystic Ovarian Syndrome (PCOS) which is one of the emerging causes of morbidity in the world.

The major criteria of PCOS, proposed in the consensus of National Institute of Health (NIH) in Bethesda, ND, USA were as follows-

a. Hyperandrogenism

b. Oligo-ovulation

c. Exclusion of other known disorder and possible the characteristic morphology of PCOS on ultrasound [1]

PCOS is set of symptoms due to elevated androgen in women.[2,3]. It is due to combination of genetic and environmental factors [4].It is the most common endocrine disorder among women between the age of 18 and 44 years [5]. It affects approximately 2- 20\% of this age group depending on how it is defined [6, 7]. It is one of the leading causes of poor fertility [2]. The earliest known description of what is now recognized as PCOS from 1721 in Italy [8]. Risk factor includes obesity, not enough physical exercise, and a family history of someone with the condition [7]. PCOS has no cure [9]. Treatment may involve lifestyle changes such as weight loss and physical exercise. Birth control pills, metformin and antiandrogen may help.

In PCOS women, normal gonadotrophin ovarian axis is disturbed, this is reflected by the higher level of LH, Lower FSH level, reversal of LH:FSH ratio. FSH level in PCOS show lower than normal values and comparable at different age of menstrual cycle without significant variation. Andgrogen dynamics involving both testosterone and Dehydro-epiandrostenidione sulphate (DHEAS) and its relation with gonadotrophin is significant in overweight/obese PCOS. Hormonal milieu in normal weight and overweight/obese women differs.

\section{Material and Methods}

Present study was carried in Rajendra Institute of Medical Sciences, Ranchi. 102 cases were studied. Study subjects were selected randomly from patient who were visiting the OPD and indoor of various department viz. Obstetrics and Gynaecology, Dermatology and Medicine.

Inclusion criteria - the diagnosis of PCOS is fulfilled by Rotterdam ESHRE/ASRM sponsored PCOS consensus criteria, when two of the following first three clinical features will be present.

a. Clinical or biochemical evidence of hyperandrogenism. 
b. Chronic anovulation

c. Polycystic ovaries on imaging

Age of study subjects were between 14 to 45 years. They readily agreed to participate in the study with an informed consent. Serum LH, FSH was evaluated with the help of chemiluminescence ABBOTT 1000 isr. Blood was collected on $3^{\text {rd }}$ day of either normal or induced menstrual cycle. LH and FSH value was evaluated from the serum. Expected value of LH during follicular and luteal phase $5 \mathrm{IU} / \mathrm{L}$ and during ovulation $60 \mathrm{IU} / \mathrm{L}$. Expected value of FSH during Follicular and luteal phase is $10 \mathrm{IU} / \mathrm{L}$ and during ovulation it is $15-20 \mathrm{IU} / \mathrm{L}$. Correlation between various parameters were analysed by Fischer's test $<0.05$ is considered significant.

\section{Observation and Results}

Table No.1 Distribution Of Cases According To Serum LH

\begin{tabular}{|l|l|l|}
\hline Serum LH & Number Of Subjects & Percentage \\
\hline Follicular Phase $>5 \mathrm{Iu} / \mathrm{L}$ & 80 & 78.43 \\
\hline Folliclular Phase $<5 \mathrm{Iu} / \mathrm{L}$ & 22 & 21.56 \\
\hline Total & 102 & 100 \\
\hline
\end{tabular}

$78.43 \%$ of patients had higher level of serum $\mathrm{LH}$

Table No.2 Distribution Of Cases According To Serum FSH

\begin{tabular}{|l|l|l|}
\hline Serum FSH & Number Of Subjects & Percentage \\
\hline$>10 \mathrm{Iu} / \mathrm{L}$ & 28 & 27.45 \\
\hline$<10 \mathrm{iu} / \mathrm{L}$ & 74 & 72.54 \\
\hline Total & 102 & 100 \\
\hline
\end{tabular}

72.54\% patients had low FSH level.

Table No.3 Distribution of Cases According To LH: FSH Ratio

\begin{tabular}{|l|l|l|}
\hline LH:FSH & Number of subjects & percentage \\
\hline$<2: 1$ & 26 & 25.49 \\
\hline$>2: 1$ & 76 & 74.50 \\
\hline total & 102 & 100 \\
\hline
\end{tabular}

$74.50 \%$ of the study group had high LH: FSH ratio.

Table no. 4 Comparison of mean serum LH, FSH levels and LH: FSH ratio in obese and non obese PCOS patients.

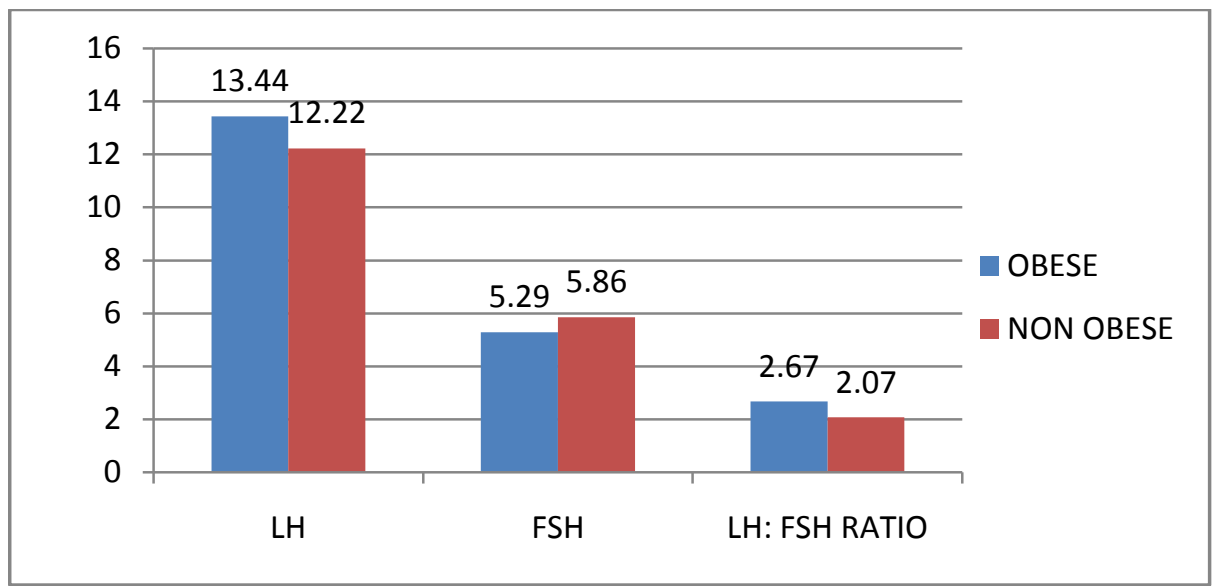

LH level is high and FSH level is low in obese subjects.

\section{Discussion}

Although PCOS is a relatively common disorder with $5-10 \%$ of prevalence among women of reproductive age its aetiology remains unknown [10]. In our study the basic finding on which diagnosis of PCOS was made in the presence of 'Polycystic ovary by ultrasound with clinical finding of oligoamenorrhoea and or hirsuitism fulfilling Rotterdam ESHRE/ASRM sponsored PCOS consensus criteria [11].

Serum level of androgens including androstenedione and testosterone may be elevated [12]. Dehydroepiandrosterone sulphate (DHEAS) level above $700-800 \mu / \mathrm{dl}$ are highly suggestive of adrenal dysfunction because DHEAS is made exclusively by the adrenal gland $[13,14]$. The ratio of LH to FSH is elevated in women with PCOS. High LH / FSH ratio are 2:1[15] or 3:1[14] as tested on day three of menstrual cycle. A ratio of $2: 1$ or higher was present in $<50 \%$ of women with PCOS in one study [15]. Antimullerian hormone $(\mathrm{AMH})$ is increased in PCOS and may become part of its diagnostic criteria [16].

DOI: $10.9790 / 0853-1601012023 \quad$ www.iosrjournals.org $21 \mid$ Page


LH/FSH ratio is not characteristic attribute of all PCOS women. Most of the PCOS women with normal gonadotrophin ratio belongs to a group of patients suffering from hyperinsulinemia and obesity [15].

There is no cure for Polysystic Ovary Syndrome and it does not go away on its own. Treatment is aimed at reducing its symptoms and preventing further complications. Options depend on the type and severity of the individual woman's symptoms and her desire to become pregnant. Diet, exercise and maintaining a healthy body weight may help many women manage the symptoms of PCOS. These life style changes are recommended to help decrease insulin resistance. Weight reduction can also decrease testosterone, insulin and LH levels.

The characteristic increase in LH relative to FSH release, have long been appreciated in PCOS. Because of the pulsatile nature of their release a single test fails to detect an increase LH/FSH ratio $[17,18]$.

Abnormality of hypothalamo- Pituitary ovarian or adrenal axis has been implicated in PCOS. Disturbance in the pulsatility of Gonadotrophin releasing hormone $(\mathrm{GnRH})$ results in the relative increase in LH to FSH release [19]. An abnormal feedback mechanism by ovarian estrogen is blamed to play role in this discriminated increase in LH release [20].

As a result of the derangement the ratio between FSH and LH level which is normally around 2 to 1 becomes reversed and sometimes even more in approximately $60 \%$ of patients with PCOS [21]. On the other hand Cho LW et al [22] found that LH/FSH ratio has little use in diagnosing PCOS because the median LH/FSH ratio did not suffer significantly between the PCOS and non affected group.

The present study was done to determine the prevalence of increased LH: FSH ratio in obese and nonobese patients. It is a corelation, clinical and biochemical single group study with 102 patients. Patients were diagnosed as PCOS using Rotterdam ESHRE/ ASRM sponsored PCOS consensus criteria.

Patients in younger age group < 20 years of age presented most commonly with acne, hirsuitism and poor fertility. Patients $>30$ years of age most commonly consulted for infertility while very few patients presented with metabolic syndrome.

Normal reproductive hormonal cycle in woman is characterized by fluctuating gonadal hormonal level, well regulated by hypophyseal-pituitary-gonadal axis. This is replaced by a relatively steady state of gonadotrophin associated with persistent anovulation.

Our study shows that in Indian PCOS women high LH: FSH ratio is a common occurrence. The mean FSH level is low as compared to normal values given for any day of menstruation and LH: FSH ratio is raised [23]. The GnRH pulse frequency designates the preferential production of LH via high frequency pulse versus FSH via low frequency pulse in normal adult women. The pulse frequency is regulated by progesterone in presence of estradiol such that increased progesterone production by corpus luteum slows LH pulse frequency to favour FSH production which aids in follicular development for the next menstrual cycle. Women with PCOS have abnormally rapid LH pulse with reduced response to progesterone feedback contributing to elevations in LH: FSH ratio [24]. LH pulse frequency was accelerated in both lean and obese PCOS, whereas the mean 24 hours LH pulse amplitude was increased in lean but not in obese PCOS patients [25].

In our study the positive correlation of LH with FSH and LH: FSH ratio is highly significant in overweight / obese women. Decreased level of FSH in the PCOS in present study indicates persistence of this negative feedback to such an extent that not a single follicle is permitted to mature enough for ovulation to occur. Result of our present study shows the possible hormonal imbalance in the form of abnormal LH and FSH secretion underlying the complex endocrinological cascade of PCOS.

\section{Conclusion}

In PCOS women, normal gonadotrophin axis is disturbed. This is reflected by high level of LH, low FSH levels and reversal of LH: FSH ratio. FSH levels in PCOS show lower than normal value and are comparable at different days of menstrual cycle without significant variations. Hormonal milieu in normal weight and overweight/obese women differs. Excessive body weight in PCOS hastens or exacerbates the complications of the disease. Hence patients of PCOS should have modification in their life style.

\section{Bibliography}

[1]. Holte, Jan, et al. "The independent effects of polycystic ovary syndrome and obesity on serum concentrations of gonadotrophins and sex steroids in premenopausal women." Clinical endocrinology 41.4 (1994): 473-481.

[2]. Polycystic ovarian syndrome (PCOS): Condition information http://www. Nichd.nih.gov/ 2013-05-23. Retrieved 13 march 2015.

[3]. Polycystic ovarian syndrome (PCOS) fact sheet women health. December 23, 2014. Retrieved 11 August 2016.

[4]. Diamanti-Kandarakis, Evanthia, Helen Kandarakis, and Richard S. Legro. "The role of genes and environment in the etiology of PCOS." Endocrine 30.1 (2006): 19-26.

[5]. Teede, Helena, Amanda Deeks, and Lisa Moran. "Polycystic ovary syndrome: a complex condition with psychological, reproductive and metabolic manifestations that impacts on health across the lifespan." BMC medicine 8.1 (2010): 41.

[6]. How many people are affected or are at risk for PCOS? http:// www.nichd.nih.gov.2013-05-23. Retrieved 13 March 2015.L Pal (2013). 'Diagnostic criteria and epidemiology of PCOS” Polycystic Ovarian Syndrone current and emerging concept. Springer P7.

[7]. Lunha Pal ( 2013 ) . " Diagnostic criteria of epidemiology of PCOS “ Polycustic ovary syndrome current and emerging concept . Dordrecht : Springer PT . 
[8]. Koracs , Gabor T, Norman, Robert ( 2007 - 2 -22 ). Polycystic ovarian syndrome Cambridge university Press P4 . Retrieved 29 March 2013

[9]. http://www.nichd.nih.gov. is there a cure for pcos? 2013-05-23. Retrieved 13 March 2015.

[10]. Dunaif, Andrea. "Insulin resistance and the polycystic ovary syndrome: mechanism and implications for pathogenesis $1 . "$ Endocrine reviews 18.6 (1997): 774-800.

[11]. ESHRE, The Rotterdam, and ASRM-Sponsored PCOS Consensus Workshop Group. "Revised 2003 consensus on diagnostic criteria and long-term health risks related to polycystic ovary syndrome." Fertility and sterility 81.1 (2004): 19-25.

[12]. Teede, Helena, Amanda Deeks, and Lisa Moran. "Polycystic ovary syndrome: a complex condition with psychological, reproductive and metabolic manifestations that impacts on health across the lifespan." BMC medicine 8.1 (2010): 41.

[13]. Somani, Najwa, Shannon Harrison, and Wilma F. Bergfeld. "The clinical evaluation of hirsutism." Dermatologic therapy 21.5 (2008): 376-391.

[14]. Polycystic ovary syndrome work up 25 October 2011. Retrieved 19 November 2011.

[15]. Banaszewska, B., et al. "Incidence of elevated LH/FSH ratio in polycystic ovary syndrome women with normo-and hyperinsulinemia." Rocz Akad Med Bialymst 48.1 (2003): 131-4.

[16]. Dewailly, Didier, et al. "The physiology and clinical utility of anti-Müllerian hormone in women." Human reproduction update 20.3 (2014): 370-385.

[17]. Dunaif A, Giniens JR , Hastline Fetal Polycystic ovarian syndrome 1992 , Blackwell scientific Cambridge MA .

[18]. Dunaif, Andrea. "Insulin resistance and the polycystic ovary syndrome: mechanism and implications for pathogenesis $1 . "$ Endocrine reviews 18.6 (1997): 774-800.

[19]. Yen SSThe polycystic ovary syndrome. Clin Endocrinol (Oxf) 1980; 12:177-207

[20]. McKenna, T. Joseph. "Pathogenesis and treatment of polycystic ovary syndrome." New England Journal of Medicine 318.9 (1988): 558-562.

[21]. Richard SL, Ricardo A. Androgen excess disorders. Danforth's obstetric and gynaecology $8^{\text {th }}$ edition, Lipincott Williams and Williams Philadelphia 2003, Chapter 37: 663-672.

[22]. Cho, Li Wei, et al. "The LH/FSH ratio has little use in diagnosing polycystic ovarian syndrome." Annals of clinical biochemistry 43.3 (2006): 217-219.

[23]. CHANG, R. JEFFREY, et al. "Insulin Resistance in Nonobese Patients with Polycystic Ovarian Disease*." The Journal of Clinical Endocrinology \& Metabolism 57.2 (1983): 356-359.

[24]. Pastor, Carmen L., et al. "Polycystic Ovary Syndrome: Evidence for Reduced Sensitivity of the Gonadotropin-Releasing Hormone Pulse Generator to Inhibition by Estradiol and Progesterone 1." The Journal of Clinical Endocrinology \& Metabolism 83.2 (1998): 582-590.

[25]. Morales, A. J., et al. "Insulin, somatotropic, and luteinizing hormone axes in lean and obese women with polycystic ovary syndrome: common and distinct features." The Journal of Clinical Endocrinology \& Metabolism 81.8 (1996): 2854-2864. 